\title{
ASYMPTOTIC VALUES AND ANGULAR LIMITS OF QUASIREGULAR MAPPINGS OF A BALL
}

\author{
SEPPO RICKMAN
}

\section{Introduction}

If $f$ is a bounded analytic function of the unit disk and $f$ has an asymptotic limit $\alpha$ at a boundary point $b$, then a wellknown theorem by E. Lindelöf says that $f$ has the angular limit $\alpha$ at $b$, and hence $\alpha$ is the only asymptotic value $f$ can have at $b$. In this paper we shall study the situation in dimensions $n \geqq 3$ for the natural generalization of analytic functions to Euclidean $n$-space, namely quasiregular mappings (for the definition, see [2]). It turns out that, although a radial limit implies the existence of an angular limit, a limit along a 1-dimensional set, like a path, is in general no longer sufficient to guarantee the existence of the angular limit, and hence Lindelöf's theorem is not true. In fact, there can be infinitely many asymptotic values at a boundary point as Theorem 1.1 shows. For the dimension $n=4$ we prove (Theorem 1.2) that here paths can as well be replaced by curved 2-dimensional half planes which have a common edge. On the other hand, Theorem 1.3 shows that $n-2$ is the maximum dimension for such results and gives a substitute for Lindelöf's theorem in a form where the asymptotic path is replaced by an $(n-1)$ dimensional set.

Let $B^{n}$ be the unit ball in the Euclidean $n$-space $R^{n}$ and $f: B^{n} \rightarrow B^{n}$ a quasiregular mapping. As we said above, if the radial limit

$$
\alpha=\lim _{t \rightarrow 1-} f(t y)
$$

exists for some $y$ in the boundary $\partial B^{n}$, then the angular limit

$$
\lim _{x \rightarrow y, x \in K} f(x)
$$

exists and equals $\alpha$ for all cones $K=K(y, \varphi)=\left\{x \in R^{n}|(y \mid(y-x))>| y-x \mid \cos \varphi\right\}$, $0<\varphi<\pi / 2[1,5.8]$. Here $(u \mid v)$ is the inner product of $u$ and $v$ in $R^{n}$. In this situation we say that $f$ has the angular limit $\alpha$ at $y$.

1.1. Theorem. For each $n \geqq 3$ there exists a quasiregular mapping $f: B^{n} \rightarrow B^{n}$ and a point $b \in \partial B^{n}$ such that 
(1) $f$ has infinitely many asymptotic values at $b$,

(2) $f$ has no angular limit at $b$.

In dimension 4 we even have a stronger result.

1.2. Theorem. There exists a sequence $U_{1}, U_{2}, \ldots$ of 2-dimensional submanifolds in $B^{4}$ such that the projection $P: R^{4} \rightarrow R^{2}, P(x)=\left(x_{1}, x_{3}\right)$, maps each $U_{j}$ homeomorphically onto the half disk $\left\{y \in R^{2}|| y \mid<1 / 2, y_{1}>0\right\}, \quad \bar{U}_{j} \cap \partial B^{4}$ is the arc $\left\{x \in \partial B^{4}\left|x_{1}=x_{4}=0,\right| x_{3} \mid \leqq 1 / 2, x_{2}<0\right\}$ for all $j$, and the following holds:

(1) Given any sequence $a^{1}, a^{2}, \ldots$ in $\left\{x \in R^{4}|| x \mid<1 / 2\right\}$ there exists a quasiregular mapping $f: B^{4} \rightarrow B^{4}$ for which

$$
\lim _{x \rightarrow-e_{2}, x \in U_{j}} f(x)=a^{j} .
$$

(2) $f$ has no angular limit at $-e_{2}$. ( $e_{i}$ is the $i^{\text {th }}$ standard coordinate unit vector in $R^{n}$.)

It seems likely that a construction can be carried out also for $n \geqq 5$ similar to the one used in the proof of Theorem 1.2 to produce an infinite number of asymptotic limits along $(n-2)$-dimensional curved half planes. However, the given proof depends on a certain 3-complex whose construction cannot canonically be generalized to higher dimensions.

Our positive result on the existence of angular limits is as follows.

1.3. Theorem. Let $n \geqq 2, A=\left\{y \in \partial B^{n} \mid y_{n}>0\right\}$, and let $f: B^{n} \rightarrow B^{n}$ be a quasiregular mapping such that for some $\alpha \in \bar{B}^{n}$

$$
\lim _{y \rightarrow e_{1}, y \in A} \limsup _{x \rightarrow y}|f(x)-\alpha|=0 .
$$

Then $f$ has the angular limit $\alpha$ at $e_{1}$

The proof of Theorem 1.3 is a simple consequence of a two constant theorem for quasiregular mappings ([7]), which is based on estimates on solutions of quasilinear elliptic partial differential equations due to Maz'ja. The proofs of Theorems 1.1 and 1.2 are rather complicated. The basic idea is to use a method developed in [6] to deform a map through adjacent cylinders into a prescribed behavior. Since $n \geqq 3$, it is possible to place the "cylinders" so that their sizes tend to zero when they approach a part of the boundary.

1.4. Remarks. 1. The proofs of Theorems 1.1 and 1.2 can be modified so that the asymptotic limits form any given countable set in the closed unit ball. One can also obtain some related results on boundary behavior, for example from the proof of Theorem 1.2 the following: There exists a nonconstant quasiregular mapping $f: B^{4} \rightarrow B^{4}$ such that

$$
\lim _{x \rightarrow y} f(x)=c=\text { constant }
$$

for $y$ in the topological disk $\left\{z \in \partial B^{4}|| z+e_{2} \mid<1, z_{4}=0\right\}$. 
2. The Lindelöf's theorem quoted above holds for quasiregular mappings for $n=2$. Theorem 1.3 reduces to a special case of this if $n=2$.

3. Because of the connection of quasiregular mappings to solutions of quasilinear elliptic partial differential equations (see [7]), our constructions give examples for such solutions too.

4. The existence of angular limits of quasiregular mappings has been studied also by $M$. Vuorinen in [8-11]. For example he has shown that for closed quasiregular mappings of $B^{n}$ into itself an asymptotic limit at $b \in \partial B^{n}$ implies the existence of the same angular limit at $b$, i.e. Lindelöf's theorem holds for these mappings.

\section{Construction of the examples}

2.1. In the proofs of Theorems 1.1 and 1.2 we shall use a construction similar to $[6, \mathrm{pp} .542-546]$. The presentation is selfcontained, hence no reference to [6] is needed.

We denote by $B^{n}(x, r)$ the open ball in $R^{n}$ with center $x$ and radius $r$, by $q$ the spherical chordal metric in $\bar{R}^{n}=R^{n} \cup\{\infty\}$, and by $\left\langle a_{1}, \ldots, a_{p}\right\rangle$ the convex hull of $\left\{a_{1}, \ldots, a_{p}\right\}$. For $1 \leqq k<n$, the sets $R^{k}$ and $R^{k} \times\{0\} \subset R^{n}$ are identified by the embedding $\left(x_{1}, \ldots, x_{k}\right) \mapsto\left(x_{1}, \ldots, 0, \ldots, 0\right)$. We set

$$
\begin{gathered}
H_{i}=\left\{x \in R^{n} \mid x_{i}>0\right\}, \quad H_{i_{1}, \ldots, i_{k}}=H_{i_{1}} \cap \ldots \cap H_{i_{k}}, \\
I_{\varepsilon}=[-\varepsilon, \varepsilon] \quad \text { for } \varepsilon>0, \quad I=I_{1}, \quad B^{n}(r)=B^{n}(0, r), \quad B^{n}=B^{n}(1), \quad S^{n-1}=\partial B^{n} .
\end{gathered}
$$

All topological operations are with respect to $\bar{R}^{n}$ if not otherwise stated.

2.2. Proof of Theorem 1.1. Set

$$
\begin{aligned}
X & =[0,1] \times] 0,1] \subset R^{2}, \\
A^{*} & =\left\{x \in R^{n} \mid\left(x_{1}, x_{2}\right) \in A,-x_{2}<x_{3}, \ldots, x_{n}<x_{2}\right\} \quad \text { if } A \subset X .
\end{aligned}
$$

We shall construct a quasimeromorphic mapping (see [3]) of int $X^{*}$ omitting a ball and with infinitely many asymptotic values at 0 . The construction is made separately in sets $C^{*}, C$ running over the set $\gamma$ of all (closed) 2-simplexes in the locally finite simplicial 2-complex $L$ with underlying space $X$ as shown in Figure 1.

Fix $C_{0} \in \gamma$ and an affine map $h_{C_{0}}$ of $C_{0}$ onto the 2-simplex $W_{1}=\left\langle-e_{1}, e_{1}, e_{2}\right\rangle \subset R^{2}$. Then there exists a unique set of affine homeomorphisms $h_{C}: C \rightarrow W_{1}$ such that $h_{C}\left|C \cap D=h_{D}\right| C \cap D$ for $C, D \in \gamma$. This follows because each vertex of $L$ in $X \backslash \dot{X}$ belongs to an even number of 2-simplexes of $L$ where $\dot{X}$ is the boundary of $X$ in $R^{2}$.

Let $W$ be the closed square $\left\langle-e_{1},-e_{2}, e_{1}, e_{2}\right\rangle \subset R^{2}$, for $0<\varepsilon<1$ let $w: W \times I_{\varepsilon}^{n-3} \rightarrow$ $\bar{B}^{n-1}$ be the radial stretching (for $n=3$ we identify $W=W \times I_{\varepsilon}^{n-3}$ ), and let $\psi_{1}=$ $w \times \mathrm{id}_{I}:\left(W \times I_{\varepsilon}^{n-3}\right) \times I \rightarrow \bar{B}^{n-1} \times I$. We choose $\varepsilon$ so that $w\left(\dot{W} \times I_{\varepsilon}^{n-3}\right) \subset \bar{B}^{n-1} \cap$ $\left(R^{2} \times I_{\varepsilon_{1}}^{n-3}\right)$ where $\varepsilon_{1}=(2(n-3))^{-1 / 2}$. We let $\varphi_{1}: \bar{B}^{n-1} \times I \rightarrow \bar{H}_{n}$ be the mapping $\varphi_{1}\left(r, y, x_{n}\right)=(\varrho, y, \theta)$ defined by $\varrho=4 e^{x_{n}+1}, \theta=\pi r / 2$. Here cylindrical and spherical 


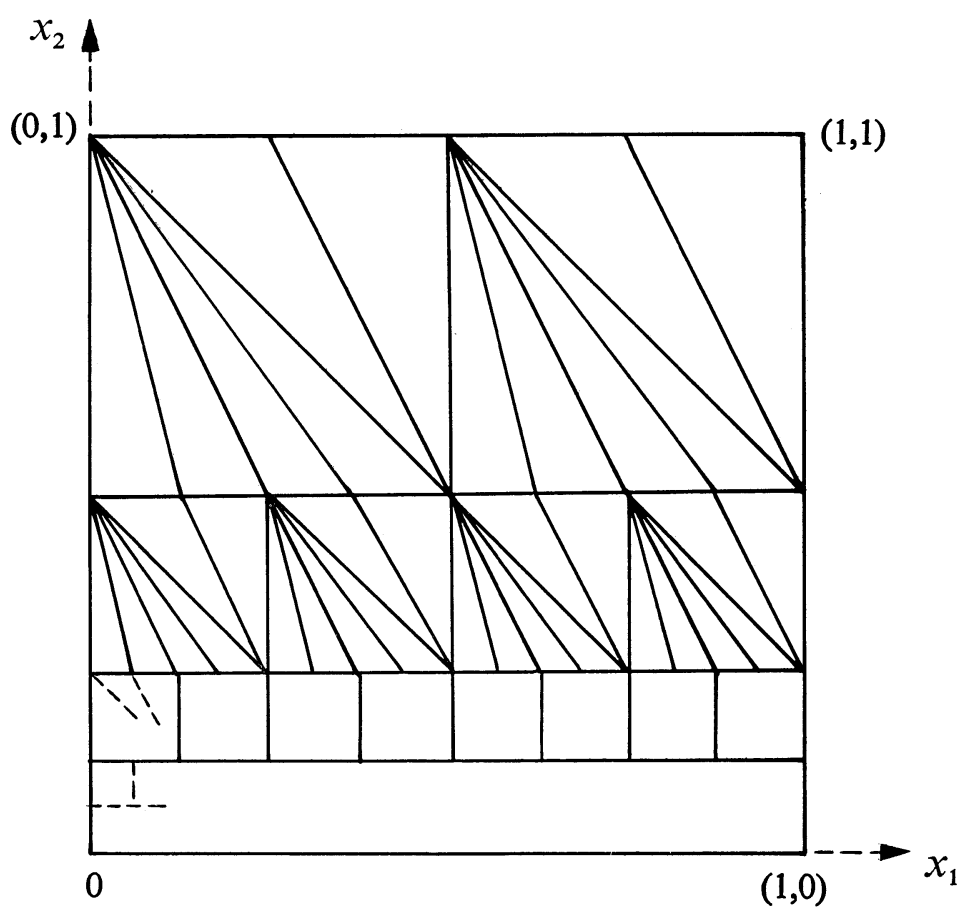

Figure 1

coordinates are used so that $y \in S^{n-2}$ and $\theta$ is the angle between the radius vector and the $x_{n}$-axis. Set $F=\varphi_{1} \circ \psi_{1}$. Then $F \mid \operatorname{int}\left(W \times I_{\varepsilon}^{n-3} \times I\right)$ is quasiconformal.

Next let

$$
\begin{aligned}
& A_{\alpha}=\left\{x \in R^{n} \mid \alpha_{i}<x_{i}<\alpha_{i}+1, i=2, \ldots, n\right\} \text { for } \alpha \in\{0\} \times Z^{n-1}, \\
& T_{1}=\left\{x \in A_{0} \mid x_{1}<-2\right\},
\end{aligned}
$$

and let $F_{1}: A_{0} \rightarrow H_{n}$ be a quasiconformal mapping such that $F_{1}(x)_{1}<0$ if $x_{1}<0$, $F_{1}(x)_{1}>0$ if $x_{1}>0, F_{1} T_{1} \subset B^{n}\left(-e_{1}, r_{0}\right)$ where we fix $r_{0}=1 / 10, F_{1}\left(A_{0} \cap B^{n}\left(4 e^{2}\right)\right) \subset$ $\bar{R}^{n} \backslash B^{n}\left(-e_{1}, r_{0} / 2\right)$, and

$$
\begin{gathered}
\lim _{x_{1} \rightarrow-\infty} F_{1}(x)=-e_{1}, \\
\lim _{x_{1} \rightarrow \infty} F_{1}(x)=e_{1} .
\end{gathered}
$$

We extend $F_{1}$ to a quasimeromorphic mapping $F_{0}: H_{2, n} \rightarrow \bar{R}^{n} \backslash\left\{-e_{1}, e_{1}\right\}$ by reflecting through the faces of the cylinders $A_{\alpha}$ and through $\partial H_{n}$. For $C \in \gamma$ we define $g_{C}: \bar{C}^{*} \rightarrow W_{1} \times I_{\varepsilon}^{n-3} \times I$ by

$$
g_{C}(x)=\left(h_{C}\left(x_{1}, x_{2}\right)_{1}, h_{C}\left(x_{1}, x_{2}\right)_{2}, \varepsilon x_{3} / x_{2}, \ldots, \varepsilon x_{n-1} / x_{2}, x_{n} / x_{2}\right) .
$$

Fix now $\beta \geqq \beta_{0}>1$ and $\left.M \in\right] 1,2\left[\right.$ where the bound $\beta_{0}$ will be chosen sufficiently large later. Let $\sigma$ be the hyperbolic metric in the upper half plane. Fix $1<\mu_{1}<$ 
$v_{1}$ and set

$$
\begin{aligned}
& Y_{0}=\left\{x \in X \mid x_{2} \geqq x_{1}^{\mu_{1}} \text { or } x_{2} \leqq x_{1}^{\nu_{1}}\right\}, \\
& Y_{k}=\left\{x \in X \mid \beta(k-1)<\sigma\left(x, Y_{0}\right) \leqq \beta k\right\}, \quad k=1,2, \ldots .
\end{aligned}
$$

Let $\delta \in] 0,1 / 2\left[\right.$ and $c^{1} \in \bar{R}^{n} \backslash B^{n}\left(-e_{1}, \delta\right)$. In the first step we shall construct a mapping $f_{1}: X^{*} \rightarrow \bar{R}^{n} \backslash B^{n}\left(-e_{1}, r_{1}\right)$ which is $K$-quasimeromorphic in int $X^{*}$, where $K$ and $f_{1} \mid Y_{0}^{*}$ are independent of $c^{1}$, where $r_{1}>0$ depends only on $\delta$, and for which

$$
\lim _{k \rightarrow \infty} \sup _{x \in Y_{k}^{*}}\left|f_{1}(x)-c^{1}\right|=0 \text {. }
$$

Choose a sequence $z^{0}=e_{1}, z^{1}, \ldots, z^{s}=z^{s+1}=\ldots=c^{1}$ in $\bar{R}^{n}$ such that

$$
M^{-1} \leqq \frac{q\left(z^{k},-e_{1}\right)}{q\left(z^{k+1},-e_{1}\right)} \leqq M, \quad k=0, \ldots, s,
$$

and $q\left(z^{k},-e_{1}\right) \geqq q\left(c^{1},-e_{1}\right), k=1,2, \ldots$. Also a sequence $x^{0}=0, x^{1}, x^{2}, \ldots$ is defined as follows. For $1 \leqq k \leqq s$ let $l_{k}$ be the minimizing geodesic arc from $-e_{1}$ to $z^{k}$ and let $x^{k}$ be the middle point of $l_{k}$. (If $z^{k}=e_{1}$, we let $l_{k}$ be the geodesic through 0 .) If $k>s$, we let $x^{k} \in l_{s}$ be so that $q\left(x^{k}, c^{1}\right) / q\left(x^{s}, c^{1}\right)=2^{s-k}$. Let $S_{k}$ be a Möbius transformation of $\bar{R}^{n}$ such that $S_{k}\left(-e_{1}\right)=-e_{1}, S_{k}\left(e_{1}\right)=z^{k}, S_{k}(0)=x^{k}, k=0,1, \ldots$. There exists a $K_{1}$-quasiconformal mapping $\omega_{k}: \bar{R}^{n} \rightarrow \bar{R}^{n}, K_{1}$ independent of $k$, such that

$$
\begin{aligned}
& \omega_{k}\left|B^{n}\left(-e_{1}, r_{0}\right)=S_{k}\right| B^{n}\left(-e_{1}, r_{0}\right), \\
& \omega_{k}\left|H_{1}=S_{k+1}\right| H_{1} .
\end{aligned}
$$

We need a map $\sigma_{1}: \bar{H}_{2, n} \rightarrow \bar{H}_{2, n}$ defined by

$$
\begin{array}{ll}
\sigma_{1}(x)=\left(x_{1}-x_{2}, x_{2}, x_{3}, \ldots, x_{n}\right) & \text { if } \quad 0 \leqq x_{2}<2, \\
\sigma_{1}(x)=\left(x_{1}-2, x_{2}, x_{3}, \ldots, x_{n}\right) & \text { if } \quad x_{2} \geqq 2 .
\end{array}
$$

If $u$ is a vertex of the complex $L$, we define

$$
P_{u}=\left\{h_{D}^{-1}\left(e_{1}\right) \mid u \in D, D \in \gamma\right\} .
$$

We are now in a position to be able to define for each $C \in \gamma$ the restriction to $C^{*}$ of the map $f_{1}$ to be constructed, call it $\tau_{C}$. Fix $C \in \gamma$. Denote $\xi=h_{C}^{-1}\left(-e_{1}\right)$, $\eta=h_{C}^{-1}\left(e_{1}\right), a=h_{C}^{-1}\left(e_{2}\right)$. Let $\bar{F}$ be $F_{0}$ if $h_{C}$ is sense preserving and $v \circ F_{0}$ if $h_{C}$ is sense reversing where $v$ is the reflection in $\partial H_{n}$. Set

$$
Q_{C}=\cup\left\{P_{u} \mid u \in D, \xi \in D, D \in \gamma\right\} \text {. }
$$

For sufficiently large $\beta_{0}$, independent of $C$, there exists a largest integer $k$ such that $Q_{C} \subset Y_{k} \cup Y_{k+1}$. In different cases we define $\tau_{C}$ as follows:

$$
\begin{array}{lll}
\tau_{C}=S_{k} \circ \bar{F} \circ F \circ g_{C} & \text { if } & P_{a} \subset Y_{k}, \\
\tau_{C}=S_{k} \circ \bar{F} \circ \sigma_{1} \circ F \circ g_{C} & \text { if } & \eta \in Y_{k}, P_{a} \cap Y_{k+1} \neq \emptyset, \\
\tau_{C}=\omega_{k} \circ \bar{F} \circ \sigma_{1} \circ F \circ g_{C} & \text { if } & \eta \in Y_{k+1}, P_{a} \cap Y_{k} \neq \emptyset, \\
\tau_{C}=\omega_{k} \circ \bar{F} \circ F \circ g_{C} & \text { if } & P_{a} \subset Y_{k+1} .
\end{array}
$$


We want to check that the maps $\tau_{C}$ coincide on common boundaries. It is enough to consider $D \in \gamma$ such that $C \cap D$ is a 1-simplex. Let $p$ be the largest integer such that $Q_{D} \subset Y_{p} \cup Y_{p+1}$.

Suppose first that $C \cap D=\langle\xi, \eta\rangle$. Then $p=k$. It follows that $\tau_{C}$ and $\tau_{D}$ may differ only in the appearance of the map $\sigma_{1}$ in the formulas (1)-(4). But $F \circ g_{C}\left(C^{*} \cap D^{*}\right) \subset$ $\partial H_{2}$ and $\sigma_{1}$ is the identity in $\partial H_{2}$.

Suppose next that $C \cap D=\langle\xi, a\rangle$. Also here $p=k$. We claim that $\bar{F} \circ \sigma_{1} \circ F \circ$ $g_{C}\left(C^{*} \cap D^{*}\right) \subset \bar{B}^{n}\left(-e_{1}, r_{0}\right)$. It is enough to show $\sigma_{1}(z)_{1} \leqq-2$ whenever $z \in F g_{C}\left(C^{*} \cap D^{*}\right)$. Such a point is of the form $z=\left(\varrho y_{1}, \ldots, \varrho y_{n-1}, 0\right), y_{3}, \ldots, y_{n-1} \in I_{\varepsilon_{1}},|y|=1, y_{1} \leqq 0$, $y_{2} \geqq 0,4 \leqq \varrho \leqq 4 e^{2}$. We may assume $0 \leqq z_{2} \leqq 2$, whence $\sigma_{1}(z)_{1}=z_{1}-z_{2}$. We have $y_{2} \leqq 1 / 2$ and $y_{1}^{2} \geqq 1-\varepsilon_{1}^{2}(n-3)-y_{2}^{2} \geqq 1 / 2-y_{2}^{2}$. This implies $y_{1} \leqq-1 / 2$ and $z_{1} \leqq-2$, hence $\sigma_{1}(z)_{1} \leqq-2$. In the cases (1) and (4) we have the same definition for $\tau_{D}$, so the assertion is clear. Suppose that we have (2) for $C$ and (3) for $D$. In this case we use the fact that $\omega_{k}\left|B^{n}\left(-e_{1}, r_{0}\right)=S_{k}\right| B^{n}\left(-e_{1}, r_{0}\right)$, and similarly if we have (2) for $D$ and (3) for $C$.

Finally, let $C \cap D=\langle\eta, a\rangle$. If $p=k$, the cases for $D$ coincide with those of $C$ in (1)-(4). By symmetry with respect to $C$ and $D$ it is enough to consider the case $p=k-1$. For a sufficiently large $\beta_{0}$, independent of $C$, we must have $Q_{C} \subset Y_{k}$, and we have case (1) for $C$ and case (4) for $D$ which fit together on $C^{*} \cap D^{*}$.

We have shown that there exists a map $f_{1}$ of $X^{*}$ such that $f_{1} \mid C^{*}=\tau_{C}$ for $C \in \gamma$. It follows from the definition of the maps $\tau_{C}$ that $f_{1}: X^{*} \rightarrow \bar{R}^{n} \backslash B^{n}\left(-e_{1}, r_{1}\right)$ for some $r_{1}>0$ and has the other required properties for the first step; $f_{1}$ lint $X^{*}$ has $c^{1}$ as an asymptotic value at 0 . For this we observe that

$$
\lim _{x \rightarrow 0, x \in E_{1}^{*}} f_{1}(x)=c^{1},
$$

where $E_{1}=\left\{x \in X \mid x_{2}=x_{1}^{\lambda_{1}}\right\}, \mu_{1}<\lambda_{1}<v_{1}$, and $E_{1}^{*}$ int $X^{*}$ contains paths tending to 0 .

Let then $\left(c^{j}\right)$ be any sequence in $\bar{R}^{n} \backslash B^{n}\left(-e_{1}, \delta\right)$, and let $1<\lambda_{1}<\lambda_{2}<\ldots$. If $1<\mu_{1}<\lambda_{1}<v_{1}<\mu_{2}<\lambda_{2}<v_{2}<\ldots$, we may deform $f_{1}$ in the sets $Y^{j *}, Y^{j}=$

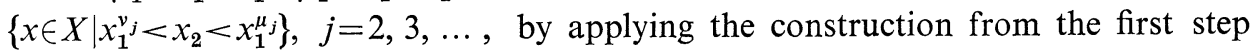
to obtain a quasimeromorphic mapping $f_{0}$ : int $X^{*} \rightarrow \bar{R}^{n} \backslash B^{n}\left(-e_{1}, r_{1}\right)$ such that

$$
\lim _{x \rightarrow 0, x \in E_{J}^{\nu}} f_{0}(x)=c^{j},
$$

where $E_{j}=\left\{x \in X \mid x_{2}=x_{1}^{\lambda_{j}}\right\}$. The required mapping satisfying (1) and (2) in Theorem 1.1 is then $f=h \circ f_{0} \circ g$ where $g$ is a suitable quasiconformal mapping of $B^{n}$ onto int $X^{*}$ and $h$ is a Möbius transformation.

2.3. Remark. The paper [6] was for simplicity written for $n=3$. The above proof shows how to do the appropriate modifications to obtain the result in [6] for general $n \geqq 3$.

2.4. Proof of Theorem 1.2. We replace the square $[0,1] \times] 0,1]$ in the proof of 1.1 by the cube $\left.\left.X^{\prime}=[0,1] \times\right] 0,1\right] \times[0,1]$ and form the locally finite simplicial 
3-complex $L^{\prime}$ as follows. In Figure 2 we let the square $\left\langle p_{0}, p_{3}, p_{2}, p_{1}\right\rangle$ be in the plane $x_{2}=1 / 2$ with $p_{0}=(0,1 / 2,0), p_{1}=(1 / 2,1 / 2,0), p_{2}=(1 / 2,1 / 2,1 / 2), p_{3}=(0,1 / 2,1 / 2)$. Let $\left\langle q_{0}, q_{3}, q_{2}, q_{1}\right\rangle$ be the square obtained from $\left\langle p_{0}, p_{3}, p_{2}, p_{1}\right\rangle$ by the translation $x \mapsto x+e_{2} / 2$, hence for example $q_{0}=(0,1,0)$. The 3 -simplexes of $L^{\prime}$ in the part $\left\langle q_{3}, p_{0}, p_{3}, p_{2}\right\rangle$ of $X^{\prime}$ are the 3 -simplexes of the form $\left\langle q_{3}, s, t, u\right\rangle$ where $\langle s, t, u\rangle$ is a 2-simplex in the 2-complex in Figure 2 with underlying space $\left\langle p_{0}, p_{3}, p_{2}\right\rangle$. The 3 -simplexes of $L^{\prime}$ in the part $\left\langle p_{0}, p_{2}, q_{3}, q_{2}, q_{0}\right\rangle$ of $X^{\prime}$ are given in Figure 3. We

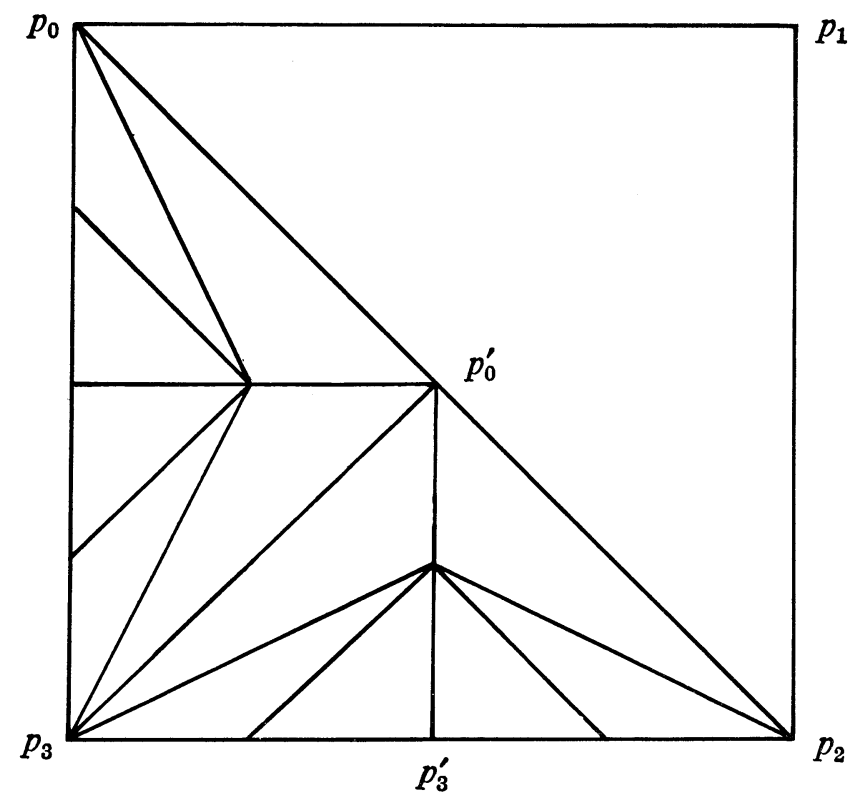

Figure 2

complete the construction of $L^{\prime}$ in the cube $\left\langle p_{0}, p_{1}, p_{2}, p_{3}, q_{0}, q_{1}, q_{2}, q_{3}\right\rangle$ by reflecting through $\left\langle p_{0}, p_{2}, q_{2}, q_{0}\right\rangle$. We continue the construction to three similar cubes with side length $1 / 2$ by first reflecting through the squares $\left\langle p_{3}, p_{2}, q_{2}, q_{3}\right\rangle$ and $\left\langle p_{1}, p_{2}, q_{2}, q_{1}\right\rangle$, and then through the square $\left\langle p_{2}, q_{2}, p_{4}, q_{4}\right\rangle$ where $p_{4}=(1 / 2,1 / 2,1), q_{4}=(1 / 2,1,1)$. We have completed the construction of $L^{\prime}$ in the layer $\left\{x \in X^{\prime} \mid 1 / 2 \leqq x_{2} \leqq 1\right\}$. The construction is continued in the layer $\left\{x \in X^{\prime} \mid 1 / 2^{2} \leqq x_{2} \leqq 1 / 2\right\}$ by a similar construction in cubes with side length $1 / 2^{2}$. Observe that for example the 2 -simplexes in $\left\langle p_{0}^{\prime}, p_{3}^{\prime}, p_{2}\right\rangle$ (Figure 2) are obtained from the 2-simplexes in $\left\langle q_{0}, q_{3}, q_{2}\right\rangle$ (Figure 3) by a similarity mapping. This gives a canonical way to continue the construction of $L^{\prime}$ to all layers $\left\{x \in X^{\prime} \mid 1 / 2^{k} \leqq x_{2} \leqq 1 / 2^{k-1}\right\}$.

Let $\gamma^{\prime}$ be the set of 3-simplexes of the complex $L^{\prime}$. Now we set

$$
A^{*}=\left\{x \in R^{4} \mid\left(x_{1}, x_{2}, x_{3}\right) \in A,-x_{2}<x_{4}<x_{2}\right\}, \quad A \subset X^{\prime} .
$$




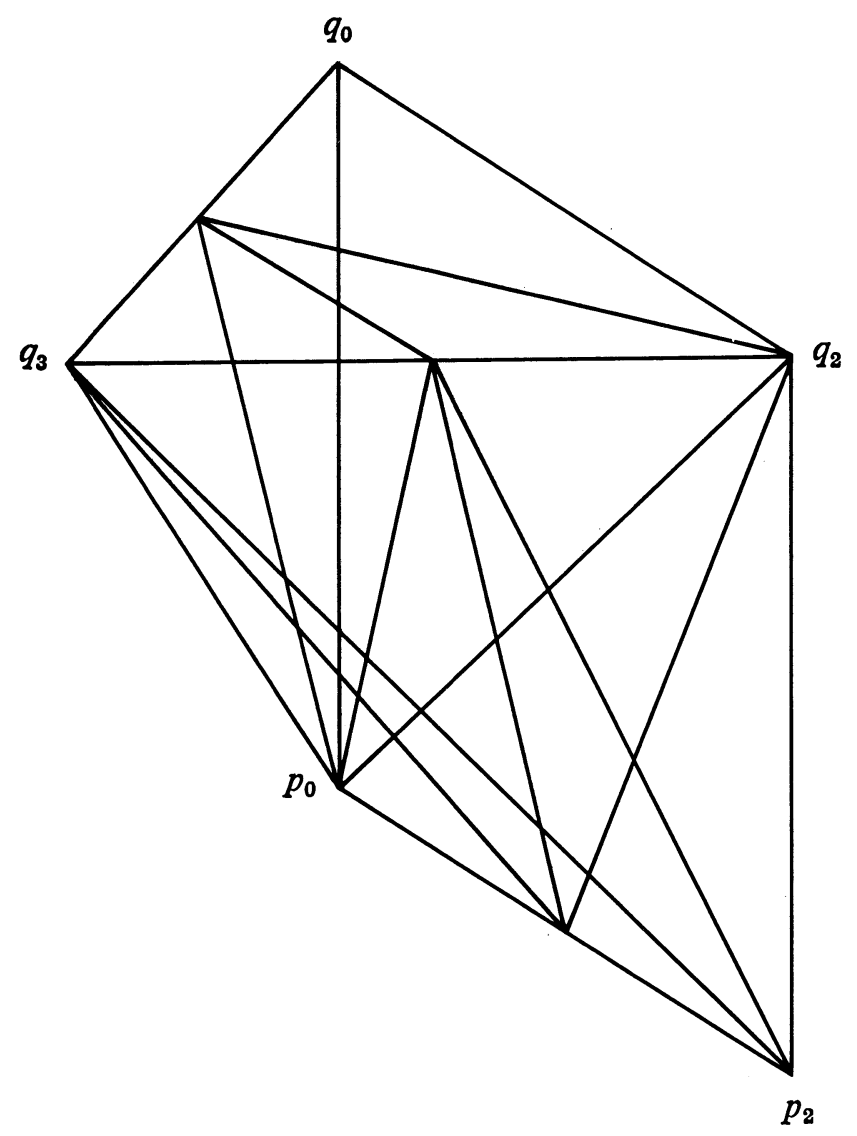

Figure 3

We fix $C_{0} \in \gamma^{\prime}$ and an affine map $h_{C_{0}}$ of $C_{0}$ onto the simplex $W_{1}^{\prime}=\left\langle-e_{1}, e_{1}, e_{2}, e_{3}\right\rangle \subset R^{3}$. It follows from the construction of $L^{\prime}$ that there exists a unique set of affine homeomorphisms $h_{C}: C \rightarrow W_{1}^{\prime}$ such that $h_{C}\left|C \cap D=h_{D}\right| C \cap D$ for $C, D \in \gamma^{\prime}$.

Let $W^{\prime}$ be the closed octahedron $\left\langle-e_{1}, e_{1},-e_{2}, e_{2},-e_{3}, e_{3}\right\rangle \subset R^{3}$, let $w^{\prime}: W^{\prime} \rightarrow \bar{B}^{3}$ be the radial stretching, and let $\psi_{1}^{\prime}=w^{\prime} \times \mathrm{id}_{I}: W^{\prime} \times I \rightarrow \bar{B}^{3} \times I$ where $I=[-1,1]$. The maps $\varphi_{1}, F_{0}$ and $\bar{F}$ are defined as in the proof of 1.1 . We set $F^{\prime}=\varphi_{1} \circ \psi_{1}^{\prime}$ and define for $C \in \gamma^{\prime} \quad g_{C}: \bar{C}^{*} \rightarrow W_{1}^{\prime} \times I$ by

$$
g_{C}(x)=\left(h_{C}\left(x_{1}, x_{2}, x_{3}\right)_{1}, h_{C}\left(x_{1}, x_{2}, x_{3}\right)_{2}, h_{C}\left(x_{1}, x_{2}, x_{3}\right)_{3}, x_{4} / x_{2}\right) .
$$

As in the proof of 1.1 let $\delta \in] 0,1 / 2\left[\right.$ and let $\left(c^{j}\right)$ be any sequence in $\bar{R}^{4} \backslash B^{4}\left(-e_{1}, \delta\right)$. Let us only consider the first step and give the definition of a map $f_{1}$ : int $X^{\prime *} \rightarrow \bar{R}^{4} \backslash B^{4}\left(-e_{1}, r_{1}\right), r_{1}>0$. The rest is similar to the end of the proof of 1.1. The sets $Y_{k}$ are defined as before, so are the Möbius transformations $S_{k}$ and the quasiconformal mappings $\omega_{k}$. 
In each $C^{*}, C \in \gamma^{\prime}$, we shall now define a map $\tau_{C}$. We need sliding maps $\sigma_{i}: \bar{H}_{2,3,4} \rightarrow \bar{H}_{2,3,4}, i=1,2,3$, defined as follows:

$$
\begin{array}{ll}
\sigma_{1}(x)=\left(x_{1}-x_{2}, x_{2}, x_{3}, x_{4}\right) & \text { if } 0 \leqq x_{2}<2, \\
\sigma_{1}(x)=\left(x_{1}-2, x_{2}, x_{3}, x_{4}\right) & \text { if } \quad x_{2} \geqq 2, \\
\sigma_{2}(x)=\left(x_{1}-x_{3}, x_{2}, x_{3}, x_{4}\right) & \text { if } 0 \leqq x_{3}<2, \\
\sigma_{2}(x)=\left(x_{1}-2, x_{2}, x_{3}, x_{4}\right) & \text { if } \quad x_{3} \geqq 2, \\
\sigma_{3}(x)=\left(x_{1}-x_{2}-x_{3}, x_{2}, x_{3}, x_{4}\right) & \text { if } 0 \leqq x_{2}+x_{3}<2, \\
\sigma_{3}(x)=\left(x_{1}-2, x_{2}, x_{3}, x_{4}\right) & \text { if } \quad x_{2}+x_{3} \geqq 2 .
\end{array}
$$

For a vertex $u$ in the 3-complex $L^{\prime}$ we set as before $P_{u}=\left\{h_{D}^{-1}\left(e_{1}\right) \mid u \in D, D \in \gamma^{\prime}\right\}$. Fix $C \in \gamma^{\prime}$ and denote $\xi=h_{C}^{-1}\left(-e_{1}\right), \eta=h_{C}^{-1}\left(e_{1}\right), a=h_{C}^{-1}\left(e_{2}\right), b=h_{C}^{-1}\left(e_{3}\right)$, and

$$
Q_{C}=\cup\left\{P_{u} \mid u \in D, \xi \in D, D \in \gamma^{\prime}\right\} .
$$

Let $\pi: R^{3} \rightarrow R^{2}$ be the orthogonal projection. For a sufficiently large $\beta_{0}$, independent of $C$, there exists a largest $k$ such that $\pi Q_{C} \subset Y_{k} \cup Y_{k+1}$.

The possible cases are as follows:

$$
\begin{aligned}
& \pi P_{a}, \pi P_{b} \subset Y_{k}, \\
& \pi P_{a} \cap Y_{k+1} \neq \emptyset, \quad \pi P_{b} \subset Y_{k}, \\
& \pi P_{a} \subset Y_{k}, \quad \pi P_{b} \cap Y_{k+1} \neq \emptyset, \\
& \pi(\eta) \in Y_{k}, \quad \pi P_{a} \cap Y_{k+1} \neq \emptyset, \quad \pi P_{b} \cap Y_{k+1} \neq \emptyset \\
& \pi P_{a} \cap Y_{k} \neq \emptyset, \quad \pi P_{b} \subset Y_{k+1}, \\
& \pi P_{a} \subset Y_{k+1}, \quad \pi P_{b} \cap Y_{k} \neq \emptyset, \\
& \pi(\eta) \in Y_{k+1}, \quad \pi P_{a} \cap Y_{k} \neq \emptyset, \quad \pi P_{b} \cap Y_{k} \neq \emptyset, \\
& \pi P_{a}, \pi P_{b} \subset Y_{k+1} .
\end{aligned}
$$

We define $\tau_{C}$ in these cases to be the following maps:

$$
\begin{aligned}
& S_{k} \circ \bar{F} \circ F^{\prime} \circ g_{C}, \\
& S_{k} \circ \bar{F} \circ \sigma_{1} \circ F^{\prime} \circ g_{C}, \\
& S_{k} \circ \bar{F} \circ \sigma_{2} \circ F^{\prime} \circ g_{C}, \\
& S_{k} \circ \bar{F} \circ \sigma_{3} \circ F^{\prime} \circ g_{C}, \\
& \omega_{k} \circ \bar{F} \circ \sigma_{1} \circ F^{\prime} \circ g_{C}, \\
& \omega_{k} \circ \bar{F} \circ \sigma_{2} \circ F^{\prime} \circ g_{C}, \\
& \omega_{k} \circ \bar{F} \circ \sigma_{3} \circ F^{\prime} \circ g_{C}, \\
& \omega_{k} \circ \bar{F} \circ F^{\prime} \circ g_{C} .
\end{aligned}
$$


Let us now check that $\tau_{C}\left|C^{*} \cap D^{*}=\tau_{D}\right| C^{*} \cap D^{*}$ for all $D \in \gamma^{\prime}$. We may suppose that $C \cap D$ is a 2-simplex. Let $p$ be the largest integer such that $\pi Q_{D} \subset Y_{p} \cup Y_{p+1}$.

Suppose first $C \cap D=\langle\xi, \eta, a\rangle$. Then $p=k$. The maps $\tau_{C}$ and $\tau_{D}$ may differ only in the appearance of the maps $\sigma_{i}$. We observe that $F^{\prime} \circ g_{C}\left(C^{*} \cap D^{*}\right) \subset \partial H_{3}$. Since $h_{D}^{-1}\left(e_{2}\right)=h_{C}^{-1}\left(e_{2}\right)=a$, all possible maps $\sigma_{i}$ coincide on $\partial H_{3}$. In fact, if the formula for $\tau_{C}$ differs from that of $\tau_{D}$, then the possible pairings are the following:
(1) for $C$ and (3) for $D$,
(2) for $C$ and (4) for $D$,
(5) for $C$ and (7) for $D$,
(6) for $C$ and (8) for $D$,

plus the interchange of $C$ and $D$. The case $C \cap D=\langle\xi, \eta, b\rangle$ is similar.

Suppose next that $C \cap D=\langle\xi, a, b\rangle$. Also here $p=k$. If the defining formulas for $\tau_{C}$ and $\tau_{D}$ are different, the only pairing which can occur is (4) for $C$ and (7) for $D$ plus the interchange of $C$ and $D$. But $\bar{F} \circ \sigma_{3} \circ F^{\prime} \circ g_{C}\left(C^{*} \cap D^{*}\right) \subset B^{4}\left(-e_{1}, r_{0}\right)$, and we use the fact $\omega_{k}\left|B^{4}\left(-e_{1}, r_{0}\right)=S_{k}\right| B^{4}\left(-e_{1}, r_{0}\right)$ to conclude that $\tau_{C}$ and $\tau_{D}$ coincide on the common part $C^{*} \cap D^{*}$.

Suppose finally that $C \cap D=\langle\eta, a, b\rangle$. If $p=k$, the cases (1)-(8) coincide for $C$ and $D$. By symmetry we may assume $p=k-1$. For a sufficiently large $\beta_{0}$, independent of $C$, we must have $\pi Q_{c} \subset Y_{k}$, and we have case (1) for $C$ and (8) for $D$. These fit together on $C^{*} \cap D^{*}$.

We have shown that there exists a map $f_{1}$ of $X^{\prime *}$ such that $f_{1} \mid C^{*}=\tau_{C}$ for all $C \in \gamma^{\prime}, f_{1}$ int $X^{\prime *}$ is $K$-quasimeromorphic $K$ not depending on $c^{1}$, and $f_{1}$ omits a ball $B^{4}\left(-e_{1}, r_{1}\right)$. For $\left.\left.A \subset X=[0,1] \times\right] 0,1\right]$ we denote now

$$
\tilde{A}=\left\{x \in X^{\prime} \mid\left(x_{1}, x_{2}\right) \in A\right\} .
$$

We observe that $f_{1} \mid \tilde{Y}_{0}^{*}$ is independent of $c^{1}$ and

$$
\lim _{x \rightarrow x_{0}, x \in \tilde{E}_{1}^{*}} f_{1}(x)=c^{1},
$$

where $x_{0}=e_{3} / 2 . E_{j}$ is as before a set $\left\{x \in X \mid x_{2}=x_{1}^{\lambda_{j}}\right\}$. This completes the first step of the proof. The construction is completed as in the proof of 1.1 to obtain a quasimeromorphic mapping $f_{0}^{\prime}$ : int $X^{\prime *} \rightarrow \bar{R}^{4} \backslash B^{4}\left(-e_{1}, r_{1}\right)$ such that

$$
\lim _{x \rightarrow x_{0}, x \in \widetilde{E}_{j}^{*}} f_{0}^{\prime}(x)=c^{j} .
$$

The required mapping is $f=h \circ f_{0}^{\prime} \circ g$ where $g: B^{4} \rightarrow$ int $X^{\prime *}$ and $h: \bar{R}^{4} \rightarrow \bar{R}^{4}$ are suitable quasiconformal mappings. The 2-manifold $U_{i}$ is the preimage by $g$ of a part of the 2-manifold $\left\{x \in \widetilde{E}_{j}^{*} \mid x_{4}=0\right\}$. The theorem is proved. 


\section{Existence of angular limits}

To prove Theorem 1.3 we need a two constant theorem for quasiregular mappings. If $f: G \rightarrow R^{n}$ is quasiregular, then the function $u(x)=\log |f(x)|$ is a solution of a quasilinear elliptic partial differential equation [5]. By using estimates for such solutions due to Maz'ja [4] we proved in [7] the following result which is a local two constant theorem.

3.1. Theorem [7, 4.22]. Let $f: G \rightarrow R^{n}$ be a quasiregular mapping of a domain $G$ in $R^{n}$, let $0<m<M$, let $U$ be a ball $B^{n}(z, \varrho)$, and suppose

(i) $|f(x)| \leqq M$ if $x \in G \cap U$,

(ii) $\limsup _{x \rightarrow y}|f(x)| \leqq m$ if $y \in \partial G \cap U$.

Then

$$
|f(x)| \leqq \exp (\beta \log m+(1-\beta) \log M) \quad \text { if } \quad x \in G \cap B^{n}(z, \gamma \varrho)
$$

where $0<\gamma \leqq \gamma_{0}$,

$$
\beta=C \operatorname{cap}\left(U, \bar{B}^{n}(z, \gamma \varrho) \backslash G\right)^{1 /(n-1)} \log (1 / \gamma),
$$

and $\gamma_{0}<1 / 2$ and $C$ are positive constants depending only on $n$ and the maximal dilatation $K(f)$ of $f$. The capacity in the expression of $\beta$ is the $n$-capacity for condensers [2].

3.2. Proof of Theorem 1.3. We shall use Theorem 3.1 in a simple iterative method. We first form a sequence $D_{1}, D_{2}, \ldots$ of subdomains of $B^{n}$ inductively by setting

$$
\begin{aligned}
& D_{1}=B^{n} \cap \bigcup_{z \in A} B^{n}\left(z, \gamma d\left(z, \partial B^{n} \backslash A\right)\right), \\
& D_{i+1}=B^{n} \cap \bigcup_{z \in D_{i}} B^{n}\left(z, \gamma d\left(z, \partial B^{n} \backslash A\right)\right),
\end{aligned}
$$

where $\gamma=\gamma_{0}$ is the constant in 3.1 and $d$ is the Euclidean metric. Let $K$ be a cone $K\left(e_{1}, \varphi\right), 0<\varphi<\pi / 2$. There exists a positive integer $k$ and $s>0$ such that $K \cap B^{n}\left(e_{1}, s\right) \subset D_{k}$. Given $\varepsilon>0$ let $\delta>0$ be such that $\delta<s$ and

$$
\limsup _{x \rightarrow y}|f(x)-\alpha|<\varepsilon \quad \text { if } \quad y \in A \cap B^{n}\left(e_{1}, \delta\right) \text {. }
$$

Then Theorem 3.1 applied to balls $B^{n}\left(z, d\left(z, \partial B^{n} \backslash A\right)\right), z \in A \cap B^{n}\left(e_{1}, \delta / 2\right)$, and to the mapping $f-\alpha$ gives

$$
|f(x)-\alpha|<e^{\beta \log \varepsilon} \quad \text { if } \quad x \in D_{1} \cap B^{n}\left(e_{1}, \delta / 3\right)
$$

where $\beta=C x^{1 /(n-1)} \log (1 / \gamma), \quad C$ being the constant in 3.1 and $x$ the capacity of the Grötzsch ring $B^{n} \backslash\left\{t e_{n} \mid 0 \leqq t \leqq \gamma\right\}$. By repeated use of this we obtain

Hence

$$
|f(x)-\alpha|<e^{\beta^{k} \log \varepsilon} \quad \text { if } \quad x \in D_{k} \cap B^{n}\left(e_{1}, \delta / 3^{k}\right) .
$$

$$
\lim _{x \rightarrow e_{1}, x \in K} f(x)=\alpha .
$$

3.3. Remark. By the method in the proof of Theorem 1.3 it is also possible to prove a global two constant theorem for general domains. 


\title{
References
}

[1] Martio, O., and S. Rickman: Boundary behavior of quasiregular mappings. - Ann. Acad. Sci. Fenn. Ser. A I 507, 1972, 1-17.

[2] Martio, O., S. RICKMAN, and J. VÄIsÄLÄ: Definitions for quasiregular mappings. - Ann. Acad. Sci. Fenn. Ser. A I 448, 1969, 1-40.

[3] Martio, O., S. Rickman, and J. VÄISÄL ̈̈: Distortion and singularities of quasiregular mappings. - Ann. Acad. Sci. Fenn. Ser. A I 465, 1970, 1-13.

[4] Maz'JA, V. G.: On the continuity at a boundary point of the solution of quasi-linear elliptic equations. - Vestnik Leningrad Univ. Math. 25 no. 13, 1970, 42-55 (Russian).

[5] RešEtnJAK, Ju. G.: Index boundedness condition for mappings with bounded distortion. Sibirsk. Mat. Ž. 9, 1968, 368-374 (Russian).

[6] Rickman, S.: A quasimeromorphic mapping with given deficiencies in dimension three. Symposia Mathematica, Istituto Nazionale di Alta Mathematica, vol. XVIII, 1976, 535-549.

[7] Rickman, S.: On the number of omitted values of entire quasiregular mappings. - J. Analyse Math. (to appear).

[8] Vuorinen, M.: Exceptional sets and boundary behavior of quasiregular mappings in $n$-space. Ann. Acad. Sci. Fenn. Ser. A I Math. Dissertationes 11, 1976, 1-44.

[9] Vuorinen, M.: On the Iversen-Tsuji theorem for quasiregular mappings. - Math. Scand. 41, 1977, 90-98.

[10] Vuorinen, M.: On the boundary behavior of locally $K$-quasiconformal mappings in space. Ann Acad. Sci. Fenn. Ser. A I 5, 1980, 79-95.

[11] VUorinen, M.: Capacity densities and angular limits of quasiregular mappings. - To appear.

\author{
University of Helsinki \\ Department of Mathematics \\ SF-00100 Helsinki 10 \\ Finland
}

Received 30 October 1979 